\title{
Contradicción no incorporada en protocolos ambientales sobre monitoreo histórico con biomonitores acuáticos como problema social
}

Contradiction not incorporated into environmental protocols on historical monitoring with aquatic biomonitors as social problem

Recibido: enero 17 de 2018 | Revisado: febrero 11 de 2018 | Aceptado: mayo 17 de 2018

\author{
George Argota Pérez \\ Yuleidis GonZÁlez PÉREZ ${ }^{2}$
}

\begin{abstract}
Resumen
Diversos protocolos ambientales solo consideran los estándares de calidad ante los límites de máximos permisibles y la concentración ya sea sobre parámetros o de elementos y en otros protocolos estandarizados utilizando organismos como bioensayos e índices sobre la toxicidad. Sin embargo, los efectos e impactos sucedidos en el tiempo mediante la interacción entre parámetros, concentraciones y organismos resultan complejo el análisis debido a limitaciones con base a programas de seguimiento. El propósito del estudio fue analizar la contradicción no incorporada en protocolos ambientales sobre monitoreo histórico con biomonitores acuáticos como problema social. Cualquier entendimiento de daños causados sobre poblaciones acuáticas debe estar sustentado en señales tempranas de predicción analítica y donde los biomarcadores en biomonitores representan, excelentes instrumentos en la toma de decisiones.
\end{abstract}

Palabras clave: monitoreo ambiental, decisiones ambientales, contradicción social, biomonitores

\begin{abstract}
Various environmental protocols only consider the quality standards to the maximum permissible limits and the concentration, whether on parameters or elements and other standardized protocols using organisms as bioassays and toxicity indexes. However, the effects and impacts that have occurred over time through the interaction between parameters, concentrations and organisms are complex due to their limitations based on monitoring programs.

The purpose of the study was to analyze the contradiction not incorporated in environmental protocols on historical monitoring with aquatic biomonitors as a social problem. Any understanding of damage caused to aquatic populations should be supported by early signals of analytical prediction and where biomarkers in biomonitors represent excellent tools in decision making.
\end{abstract}

Key words: environmental monitoring, environmental decision-making, social contradiction, biomonitors 


\section{Introducción}

Desde la aparición del hombre en la Tierra, diferentes razones de interpretación cognoscitiva se han incorporado para resolver disímiles problemas sociales de acuerdo al momento histórico (Montealegre, 2007). El desarrollo logrado en la humanidad es producto del conocimiento trans-generacional; y sin duda alguna, las contradicciones según los paradigmas de existencia, condicionan diferentes comunicaciones conceptuales para la solución gradual y contemporánea sobre los constantes problemas antropogénicos generados.

Actualmente, resulta de interés, cómo el hombre asocia su conocimiento en la evaluación ambientalmente segura sobre los cuerpos de aguas superficiales. De forma tradicional e histórica, los parámetros físico-químicos y microbiológicos de calidad del agua continúan siendo lo realizado y quizás, rutinariamente, aunque siempre habrá que reconocer, el carácter temporal de sus interpretaciones durante un momento y espacio específico donde la limitación principal radica, en carecer de información referida con posibles efectos e impactos negativos sobre algunas poblaciones por exposición a contaminantes de interés (Argota, 2017). Uno de los contaminantes de interés son los metales pesados de modo que, pudiera valorarse la siguiente interrogante: ¿cuáles podrían ser los daños próximos por exposición a metales pesados en los ecosistemas acuáticos?

Antes de analizar consecuencias probables debe comprenderse que, diversos ecosistemas acuáticos muestran gran desequilibrio ambiental lo cual significa, limitaciones como recurso (Elgallal, Fletcher \& Evans, 2016), pues diversos procedimientos tecnológicos implementados en las plantas de tratamiento, por lo general, no eliminan algunos elementos y entre ellos figuran los propios metales pesados: MP (Lee \& Dhar, 2012; Dixit et al., 2015) y por ende, los efluentes son vertidos con elevada peligrosidad, lo que resulta poca eficiencia sobre la seguridad ante cualquier valor de uso posterior. La disminuida biodegradabilidad de los MP (Qadir \& Malik, 2011; Raval, Shah \& Shah, 2016) genera bioacumulación en organismos inferiores y luego, puede biomagnificarse (Molina, Ibañez \& Gibon, 2012) causando riesgos irreparables en la salud humana (Han et al., 2016).

La predicción de riesgo en ecotoxicología acuática por exposición a MP, sigue siendo mediante comparaciones con valores referenciales regulatorios, pero la razón de que las normas constituyan valiosa herramienta como variante de extrapolación de resultados a humanos, no podrán señalar exposiciones agudas y menos de tipo crónica (Figura 1).

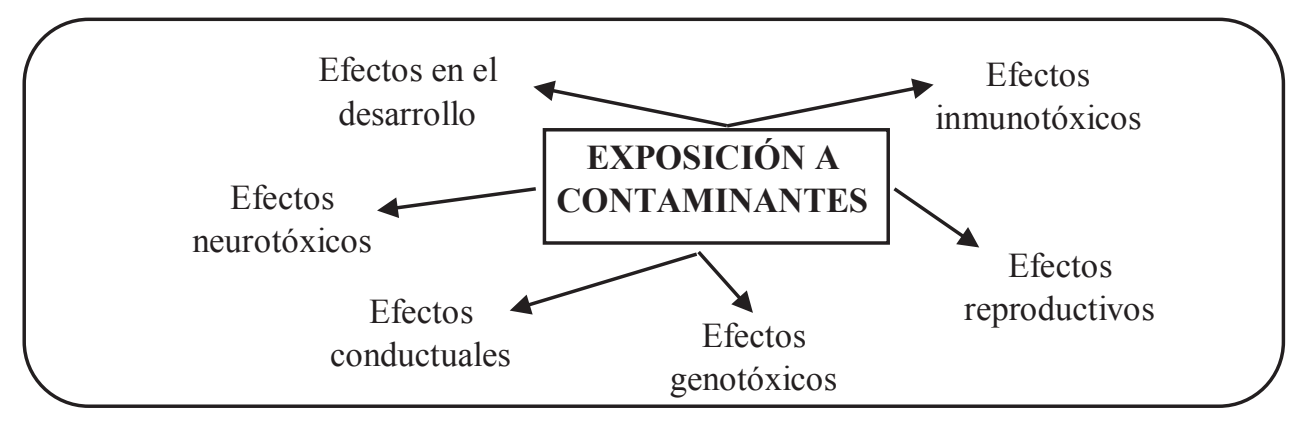

Figura 1. Efectos biológicos no deseados por exposición a contaminantes

Fuente: Argota, P.G. (2017: p.10) 
Ante tal situación ambiental, nuevos son los problemas que se plantean en la ciencia donde son admitidas las políticas identificadas para que cada ciencia trate de forma constante, indagar sobre decisiones retroalimentadas (Cazáres et al., 2006). Muchos casos carecen de respuestas únicas y completas y en consecuencia, es preciso aprender a lidiar con la complejidad, incertidumbre y el riesgo. En materia ambiental con frecuencia, no es posible predecir y explicar sobre la base de teorías probadas, pues solo resulta frecuentemente, la tenencia de modelos y/o simulaciones, generándose algunas soluciones aproximadas (Funtowicz \& Ravetz, 1997). El propósito del estudio fue analizar la contradicción, no incorporada, en protocolos ambientales sobre monitoreo histórico con biomonitores acuáticos como problema social.

\section{Desarrollo}

Al interpretar la definición de Truahut (1969), profesor e investiga- dor francés, quien sugirió por primera vez y durante una reunión celebrada en junio por el Comité del Consejo Internacional de Uniones Científicas con sede en la ciudad de Estocolmo-Suecia, la terminología "Ecotoxicología", hizo mención como: "extensión natural de la Toxicología" (ciencia que estudia los efectos de las sustancias tóxicas sobre los organismos individuales). Para el uso de la terminología, refirió indicación hacia los efectos ecológicos ocasionados por contaminantes: 1 ro) la toxicidad hacia cualquier organismo; y 2do) las alteraciones del medio en el cual viven los organismos. En este sentido, se indicó que un contaminante al provocar la muerte sobre el $50 \%$ de los individuos dentro de una determinada población, podría tener poca significación, pero si el contaminante no provoca la muerte, aunque retarda cualquier función biológica (efecto biológico no deseado) o altera el medio ambiente, sí podría tener gran implicación (Figura 2).

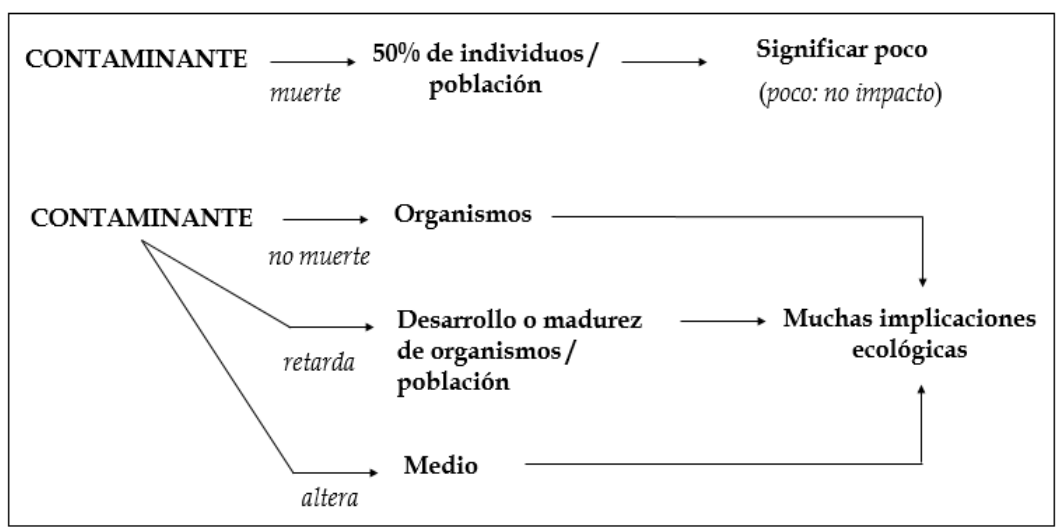

Figura 2. Interpretación del concepto de ecotoxicología propuesto por Truahut (1969)

Considerando la segunda razón de efecto ecológico puede describirse que, algunas fluctuaciones, en los parámetros físico-químicos y microbiológicos quizás fijan, respuestas biológicas, pero es poco visible su carácter inmediato. Por el con- trario, la interacción como un todo de los parámetros físico-químicos y microbiológicos, entonces condicionaría a respuestas biológicas tardías y en esta única orientación, todo esquema conductual humano a la hora de tomar decisiones 
ambientales puede conducir a incorrectas interpretaciones. ¿De qué dependería la diferencia? Naturalmente, de considerar, en forma multivariada, el comportamiento de los parámetros físico-químicos y microbiológicos, pero deberá estar sustentado en un monitoreo histórico o de vigilancia ambiental (Figura 3).

Sin embargo, la mayoría de los protocolos de monitoreo no describen las acciones a seguir ante la evidencia registrada de daños ambientales, en la columna de agua, sedimentos o las propias matrices

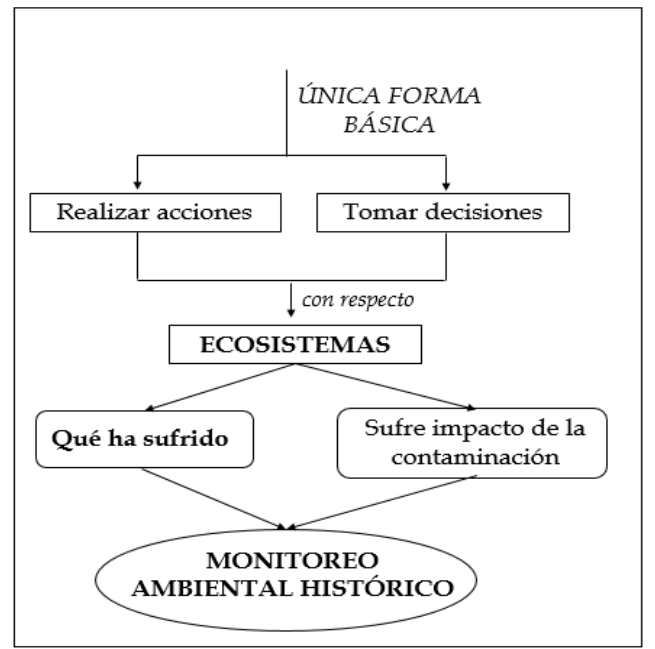

Figura 3. Monitoreo ambiental histórico Fuente: Argota (2017, p. 17)

Aunque la demostración de la contaminación ambiental resulta poco habitual, la Figura 4 permite, de manera práctica, una reflexión. La Primera Ley de la Termodinámica indica que, la energía ni se crea ni se destruye solo se conserva (transforma) y fundamentando este principio sobre los ecosistemas contaminados, su comprensión estaría en reconocer que, la probabilidad de ocurrencia con relación a un daño adverso, no siempre es baja, pues la retención sobre de la energía (materia), dentro del ecosistema acuático, puede ser más perjudicial al es- biológicas. Las evaluaciones con base al monitoreo ambiental histórico posibilitan conocer, cómo se van comportando los recursos dentro del ecosistema, además, de informar en paralelo, cualquier deterioro ambiental ocasionado por cuanto, esta información, no es otra razón que analizar el grado evolutivo de un ecosistema. Para ello, dos grandes retos científicos de demostración siempre estarán presentes: 1ro) presencia de un agente tóxico y 2do) aun en bajas concentraciones, cualquier agente puede ser tóxico y ejercer efecto dañino en la biodiversidad acuática y asociada.

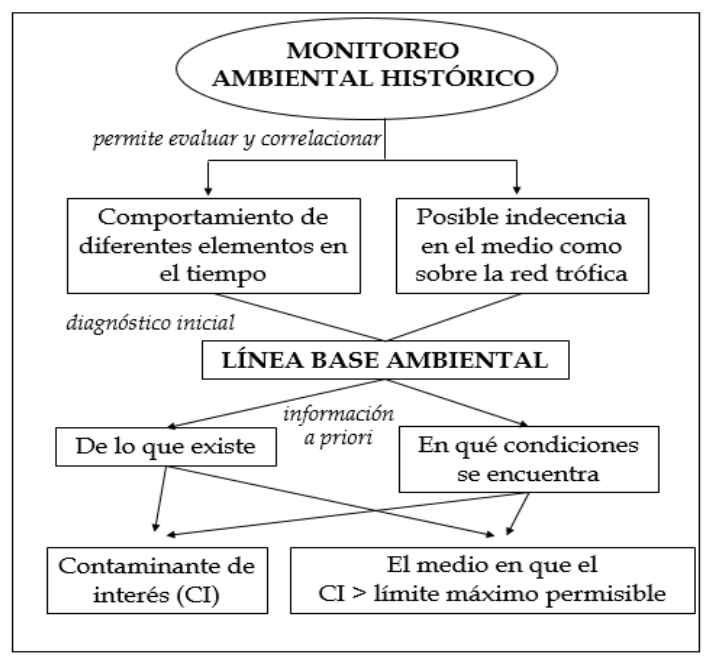

tacionarse durante un momento y periodo dado.

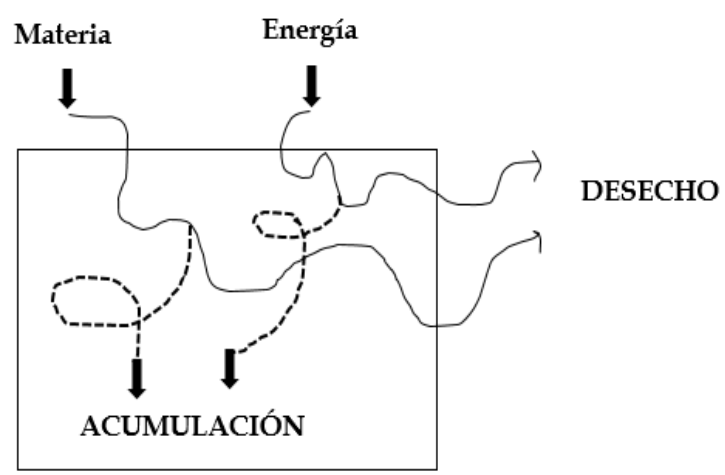

Figura 4. Principio termodinámico aplicado en un ecosistema acuático Fuente: Argota, P.G. (2017, p. 15) 
Asimismo, al medirse las fluctuaciones de los parámetros físico-químicos y microbiológicos, en el ecosistema acuático, siempre resultará complejo evaluar el impacto temporal de cualquier contaminación (Figura 5) como puede ser, la exposición a metales pesados: MP.

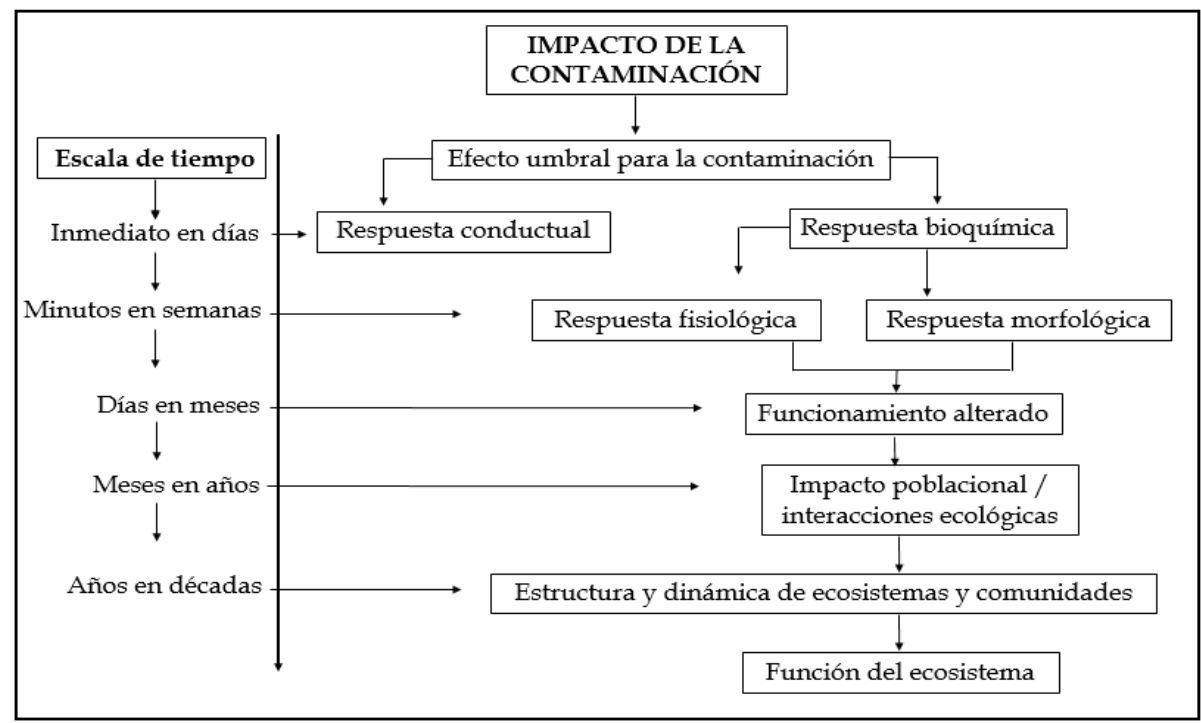

Figura 5. Escala (impacto) temporal de la contaminación sobre el ecosistema acuático

Fuente: Argota (2017, p. 16)

Ante las apreciaciones anteriores, los problemas sociales siguen siendo contradictorios, pues la medición de los parámetros físico-químicos y microbiológicos no dimensiona las respuestas ecotoxicológicas y por tanto, no arroja total seguridad ambiental de tal modo que, otras herramientas requerían ser incorporadas. En tal sentido, el uso de índices (López, Figueroa \& Corrales, 2016), creación de modelos computacionales (Chapman \& Riddle, 2003; Patlewicz \& Fitzpatrick, 2016) y análisis por bioensayos (Darrigran, Pereyra \& Rossini 2012; Fahd et al., 2014; Hedayati \& Sadeghi, 2014) se han incorporado, pero dichas herramientas de forma independiente, limitan las interpretaciones sobre la calidad ambiental y económica sostenible de los recursos acuáticos.

La medición económica y sostenible sobre la calidad ambiental ha sido abordada con diferentes perspectivas, externalidades, contingencias, derecho de propiedad, eficiencia económica y desde la razón sobre la pérdida del bienestar (Butler, Corvalan \& Koren, 2005). Algunos enfoques intentan, además, establecer conexiones teóricas entre los sistemas ecológicos y económicos (Volk et al., 2007; Jiajun et al., 2014), pero estas conexiones resultan muy complejas posiblemente por la inexistencia valorativa en muchas legislaciones internacionales con razón a la propia orientación económica (David et al., 2004; Pérez, Peńa \& Alvarez, 2011).

Durante los últimos años, la predicción ante el riesgo ecotoxicológico por exposición a MP en los ecosistemas acuáticos ha sido utilizando organismos naturales como los peces debido a considerarse, entre los primeros, en ser empleados por protocolos de evaluación ecotoxico- 
lógica (Çiftçi et al., 2015; Hamza, 2014; Heidary, Imanpour \& Monsefrad, 2011), unido a su condición de organismos centinelas para cualquier estudio ambiental (Argota, Iannacone \& Fimia, 2013; AbdAllah, 2017).

Parece incuestionable el reconocimiento de los organismos bioindicadores o biomonitores, desarrollados en ambientes naturales y donde esta última condición es más reflexiva para el análisis en ecotoxicología acuática, pero tampoco resulta simple su empleo, ya que diversos criterios son reportados para su consideración y entre ellos figuran: 1 ro) tener distribución cosmopolita, 2do) ser de fácil identificación taxonómica, 3ro) tener tamaño apropiado, 4to) presentar movilidad limitada y ciclo de vida relativamente largo, 5to) ser tolerantes a la contaminación, 6to) presentar fácil manejo para estudios de laboratorios; y $7 \mathrm{mo}$ ) debe existir una correlación entre el contaminante de interés y el resto de las matrices ambientales (Díaz, 1995; Rosenberg \& Pesh, 1993).

Tampoco los protocolos de monitoreo ambiental consideran el uso de esta clasificación y parece contradictorio, pues su aceptación por la comunidad científica internacional, es una realidad. Aunque se señaló siete criterios como condición para ser organismo biomonitor, Argota \& Iannacone (2017) señalaron que, no significan que ser únicos e invariables y para ello surgió un 8vo criterio, al cual titularon: predicción cuantitativa de riesgo histórico entre ecosistemas impactados y de referencia ambiental mediante uso permanente de biomarcadores. Este criterio sustenta la necesidad de que todo programa de monitoreo ambiental histórico debe ser implementado de forma permanente.

Finalmente, las últimas tendencias en ecotoxicología acuática están relacionadas con el uso de biomarcadores como herramientas predictivas ante la expresión de síntesis sobre determinada información cualitativa y/o cuantitativa en el tiempo indicando posibles efectos e impactos que generan los contaminantes (Argota \& Iannacone, 2017; Lock \& Bonventre, 2008) y donde los protocolos ambientales tendrían que calificar para ser más fidedignos.

\section{Referencias}

AbdAllah, A.T. (2017). Efficiency of invertebrate animals for risk assessment and biomonitoring of hazardous contaminants in aquatic ecosystem, a review and status report. Environ Risk Assess Remediat; 1(1), 22-24. http://www.alliedacademies.org/environmental-risk-assessment-and-remediation/
Argota, P.G. (2017). Monitoreo biológico ambiental en ecotoxicología acuática. Cusco, Perú: Editorial Kopy Graft E.I.R.L., 41, 10, 15, 16, 17. ISBN: 978-612-00-2558-1

Argota, P.G, Iannacone, O.J. \& Fimia, D.R. (2013). Características de Gambusia punctata (Poeciliidae) 
para su selección como biomonitor en ecotoxicología acuática en Cuba. Revista The Biologist; 11(2), 229236. http://sisbib.unmsm.edu.pe/ bvrevistas/biologist/v11_n2/pdf/ a5v11n2.pdf

Argota, P.G. \& Iannacone, O.J. (2017). Predicción cuantitativa mediante biomarcadores de uso permanente como nuevo criterio para biomonitores en ecotoxicología acuática. Revista The Biologist; 17(1), 141153.

Butler, C.D., Corvalan, C.F. \& Koren, H.S. (2005). Human health, well-being, and global ecological scenarios. Ecosystems; 8(2), 153-162. Doi: $10.1007 / \mathrm{s} 10021-004-0076-0$

Cázares, G. Y.M.; Morales, F.F.; Lozano, V.A.L. \& Camacho, L.M.I. (2006). Ética y valores 2. Un Acercamiento práctico, 354. México: Thomson.

Chapman, P.M. \& Riddle, M.J. (2003). Missing and needed: polar marine ecotoxicology. Mar Pollut Bull; 46(8), 927-928. Doi:10.1016/j. marpolbul.2003.12.008

Çiftçi, N., Ay, Ö., Karayakar, F., Cicik, B. \& Erdem, C. (2015). Effects of zinc and cadmium on condition factor, hepatosomatic and gonadosomatic index of Oreochromis niloticus. Fresenius Environmental Bulletin; 24(11A), 3871-3874.

David, J., Múnera, O., Restrepo, F.C., Económica, V., Ambientales, D.E.C. \& Conceptual, M. (2004). Costos Ambiental- es: Marco Conceptual y Métodos. Semestre Económico, 7(13), 159-193. http://www.redalyc.org/ pdf/1650/165013657006.pdf

Díaz, M.M. (1995). Biomonitoreo en sistemas lénticos: El uso de especies indicadoras. Zoología informa; 31, 17-35.

Dixit, R., Wasiullah, Malaviya, D., Pandiyan, K., Singh, U.B. \& et al. (2015). Bioremediation of heavy metals from soil and aquatic environment: An overview of principles and criteria of fundamental processes. Sustainability (Switzerland); 7(2), 2189-2212. Doi:10.3390/ su7022189

Elgallal, M., Fletcher, L. \& Evans, B. (2016). Assessment of potential risks associated with chemicals in wastewater used for irrigation in arid and semiarid zones: A review. Agric Water Manag; 177, 419-431. Doi:10.1016/j.agwat.2016.08.027

Fahd, F., Khan, F., Hawboldt, K. \& Abbassi, R. (2014). Developing a novel methodology for ecological risk assessment of thiosalts. Stochastic Environmental Research and Risk Assemente; 28(2), 383-391. Doi:10.1007/s00477-013-0758-2

Funtowicz, S. \& Ravetz, J. (1997). Problemas ambientales, ciencia post-normal y comunidades de evaluadores extendidas. En: Ciencia, Tecnología y Sociedad. Lecturas Seleccionadas. Ed. Ariel, 23-37. Barcelona. 
Hamza, C.A. (2014). Usefulness of Bioindicators and Biomarkers in Pollution Biomonitoring. International Journal of Biotechnology for Wellness Industries; 3, 19-26.

Han, W., Fu, F., Cheng, Z., Tang, B. \& Wu, S. (2016). Studies on the optimum conditions using acidwashed zero-valent iron/aluminum mixtures in permeable reactive barriers for the removal of different heavy metal ions from wastewater. I Hazard Mater; 302, 437-446. Doi:10.1016/j. jhazmat.2015.09.041

Heidary, S., Imanpour, N. \& Monsefrad, F. (2011). Bioaccumulation of heavy metals $\mathrm{Cu}, \mathrm{Zn}$, and $\mathrm{Hg}$ in muscles and liver of the stellate sturgeon (Acipenser stellatus) in the Caspian Sea and their correlation with growth parameters. Iranian Journal of Fisheries Sciences; 11(2), 325-337. http://jifro.ir/article-1-531-en.pdf

Jiajun, H., Huimin, Z. Hui, Z., Xuan, G., Mingwei, S., Junhao, Z. \& Xiaotao, L. (2014). Ecological risk and economic loss estimation of heavy metals pollution in the Beijiang River. Ecol Chem Eng S; 21(2), 189-199. Doi:10.2478/eces-20140015

Lee, J. \& Dhar, B. (2012). Bio-processing of solid wastes and secondary resources for metal extraction- A review. Waste Manag; 32, 3-18. Doi:10.1016/j.wasman.2011.08.010
López, I.D., Figueroa, A. \& Corrales, J.C. (2016). A Systematic Mapping of Water Quality Prediction Using Computational Intelligence Techniques. Revista Ingenierias Universidad de Medellin; 15(28), 35-51. Doi.10.22395/rium.v15n28a2

Lock, E.A. \& Bonventre, J.V. (2008). Biomarkers in translation; past, present and future. Toxicology; 245(3), 163-166. Doi.10.1016/j. tox.2007.12.004

Molina, C.I., Ibañez, C. \& Gibon, F.M. (2012). Proceso de biomagnificación de metales pesados en un lago hiperhalino (Poopó, Oruro, Bolivia): Posible riesgo en la salud de consumidores Biomagnification process of heavy metals of a hiperhaline lake. Ecología en Bolivia; 47(2), 99-118. http://www.scielo.org.bo/ $\mathrm{pdf} / \mathrm{reb} / \mathrm{v} 47 \mathrm{n} 2 / \mathrm{v} 47 \mathrm{n} 2 \_\mathrm{a} 03 . \mathrm{pdf}$

Montealegre, R. (2007). La solución de problemas cognitivos. Una reflexión cognitiva sociocultural. Avances en Psicología Latinoamericana; 25(2), 20-39. http://www.scielo.org.co/ $\mathrm{pdf} / \mathrm{apl} / \mathrm{v} 25 \mathrm{n} 2 / \mathrm{v} 25 \mathrm{n} 2 \mathrm{a} 3 . \mathrm{pdf}$

Patlewicz, G. \& Fitzpatrick, J.M. (2016). Current and future perspectives on the development, evaluation, and application of in silico approaches for predicting toxicity. Chem Res Toxicol;29,438-451. Doi:10.1021/ acs.chemrestox. 5 b00388

Pereyra, P.J., Rossini, G.B. \& Darrigran, G. (2012). Toxicity of Neem's oil, a potential biocide against the in- 
vasive mussel Limnoperna fortunei (Dunker 1857). Anais Da Academia Brasileira de Ciencias; 84(4), 1065-1071. Doi:10.1590/S000137652012005000059

Pérez, M.A., Peña, M.R. \& Alvarez, P. (2011). Agro Industria Cañera y uso del agua: análisis crítico en el contexto de la política de agrocombustibles en Colombia. Ambiente o Sociedade; XIV(2), 153-178. http://www.scielo.br/pdf/asoc/ v14n2/11.pdf

Qadir, A. \& Malik, R. N. (2011). Heavy metals in eight edible fish species from two polluted tributaries (Aik and Palkhu) of the river Chenab, Pakistan. Biological Trace Element Research; 143(3), 1524-1540. Doi:10.1007/s12011-011-9011-3

Raval, N.P., Shah, P.U. \& Shah, N.K. (2016). Adsorptive removal of nickel (II) ions from aqueous environment: A review. J Environ Manage; 179, 1-20. Doi:10.1016/j.jenvman.2016.04.045
Rosenberg, D.M. \& Pesh, W.H. (1993). Freshwater biomonitoring and benthic macroinvertebrates. New York: Chapman \& Hall. Pp 488.

Sadeghi, A. \& Hedayati, A. (2014). Investigation of LC50, NOEC and LOEC of Glyphosate, Deltamethrin and Pretilachlor in Guppies (Poecilia reticulata). Iranian Journal of Toxicology; 8(26), 1124-1129. http://ijt.arakmu.ac.ir/article-1338-en.pdf

Truhaut, R. (1977). Eco-toxicologyobjectives, principles and perspectives. Ecotoxicol Environ Saf; 1, 151-173.

Volk, M., Hirschfeld, J., Schmidt, G., Bohn, C., Dehnhardt, A. \& et al. (2007). A SDSS-based ecological-economic modelling approach for integrated river basin management on different scale levels - The project FLUMAGIS. Water Resources Management; 21(12), 20492061. Doi:10.1007/s11269-0079158-z 